\title{
Orientation and Development of Surveying, Mapping and Geo-Information under the Smart City Construction
}

\author{
Xitao Huang ${ }^{1,2, *}$, Huaien $\mathrm{Li}^{1}$, Lingling $\mathrm{Liu}^{2}$, Yu Zhang ${ }^{2}$ and Lijie Feng ${ }^{2}$ \\ ${ }^{1}$ State Key Laboratory Base of Eco-Hydraulic Engineering in Arid Area, Xi'an University of Technology, Xi'an, Shaanxi \\ Province P.R. China 710054 \\ 2334 Youyi East Road, Xi'an, Shaanxi Province P.R. China 710054 \\ "Corresponding author
}

\begin{abstract}
Along with Chinese urbanization and informatization has become increasingly mature, the traditional urban management problems and drawbacks exposure increased, the smart city construction as a new model of effective management, which at the same time provides a new opportunity for Surveying, Mapping and Geo-information(SMG) transformation and upgrading of spanning development. Besides analyzing the essence understanding of Smart City, the difference between Smart City and Digital city, and the construction content and key technologies of SMG, this paper also describes and analyzes how to interface SMG and informatization. Meanwhile, SMG services, construction of smart city and SMG development has carried on the beneficial exploration.
\end{abstract}

Keywords-surveying; mapping and geo-information; smart city; informatization

\section{INTRODUCTION}

To implement "the notice of CPC Central Committee and State Council on the issuance of new national urbanization plan (2014-2020)" (CPC [2014] No. 4), the State Council in 2014 issued the "guiding opinions on promoting the healthy development of the smart city". The document notes: "smart city is a kind of new ideas and new models which as a new generation of information technology integrates Geographic space, the internet of things (IOT), cloud computing, big data etc., promotes urban planning, construction, intelligent management and service. Smart city could integrate overall urban geospatial information, structures database, and other resources to accelerate the construction of intelligent urban public information platform and application system. With unified geospatial framework and demographic, legal and other resource-based information superimposed departments, and industry-related service information, accelerate the promotion of cross-sectoral collaborative applications. "That is a clear and precise definition of the smart city and roles of various sectors in the urban construction. Meanwhile, it also explains SMG as the basic resources in Smart city construction.

In recent years, NASG seize the opportunity to construct urban spatial data infrastructure in the "fifth" period. Up to the end of December 2014, 333 cities and more than 380 counties carried out the construction of digital cities, among which more than 220 cities and more than 100 counties have completed construction of digital cities and put into use. More than 3,600 applications were developed in national wide, involves in land, planning, transportation, real estate, public security, fire protection, environmental protection, health, public services and other dozens of areas.

In this important historical transition, SMG developed great strides forward, innovation, and service in order to obtain a win-win cooperation blueprint. This article will discuss SMG service mode, core technology, key applications, interfaces mode and other aspects during Digital City to Smart City, which will be a very useful exploration on Smart City implementation.

\section{The ESSENTIAL UNDERSTANDING OF SMART CiTY}

Smart is the ability of people to things fast, flexible, properly understood and solve. Smart or Wisdom is another milestone in human technology breakthrough since the industrialization, electrification, information technology. Smart City is a higher level of digital, information technology and automation, its core is to imitate human thinking, relying on "big (big data), cloud (cloud computing), shift (mobile Internet), physical (IOT)." , the transaction will be carried out between each other and consider the optimal combination of demand, through information input, processing, analysis, judgment, and ultimately concluded. Get digitized first and then information technology, multi-source information industries in different areas and phases of the integration, analysis and mining became the basis for making judgments, reuse expert knowledge system to judge concluded, and build automation, artificial information intelligent city run on the basis of the intervention. Because human wisdom is endless, so explore the wisdom of the city is endless.

\section{THE DIFFERENCE BETWEEN SMART CITY AND DIGITAL CITY}

Wisdom of urban development also follow the general rules of development, from lower to higher, from the digitized information to then intelligence. The Smart City which with the knowledge engine could self-learning, ability-enhancing, and provides intelligent services by following people's needs, intelligently promotes scientific development of the city.

For SMG departments, geospatial framework as a digital city, smart city "skeleton" has always been our focus and 
innovative positioning reference point. Geospatial framework includes GIS data collection, processing, exchange of services, as well as involved in policy, regulations, standards, technologies, facilities, mechanisms and human resources, etc. Geospatial framework consists of the basic geographic information system, catalog and exchange system, multiple structures of public service system, policies and standards, etc. Thus, it is a fundamental part of Digital City and Smart City. In digital age, geospatial framework services embodied in basic geographic information database and platform. With the development of computer, multimedia, Internet and other technologies, particularly cloud computing, networking, Internet + rapid development of technology, smart city of the existing geospatial framework put forward new and higher requirements. In smart age, its services embodied in temporal information and temporal information cloud database platform, based on the outcome of the digital city construction, development of SMG in real-time access to accurate positioning, organic integration, the use of depth, knowledge engine, on-demand service achieve to the industry in various fields, from time to time to provide various aspects, automation, fine, intelligent service, as shown in Figure I.

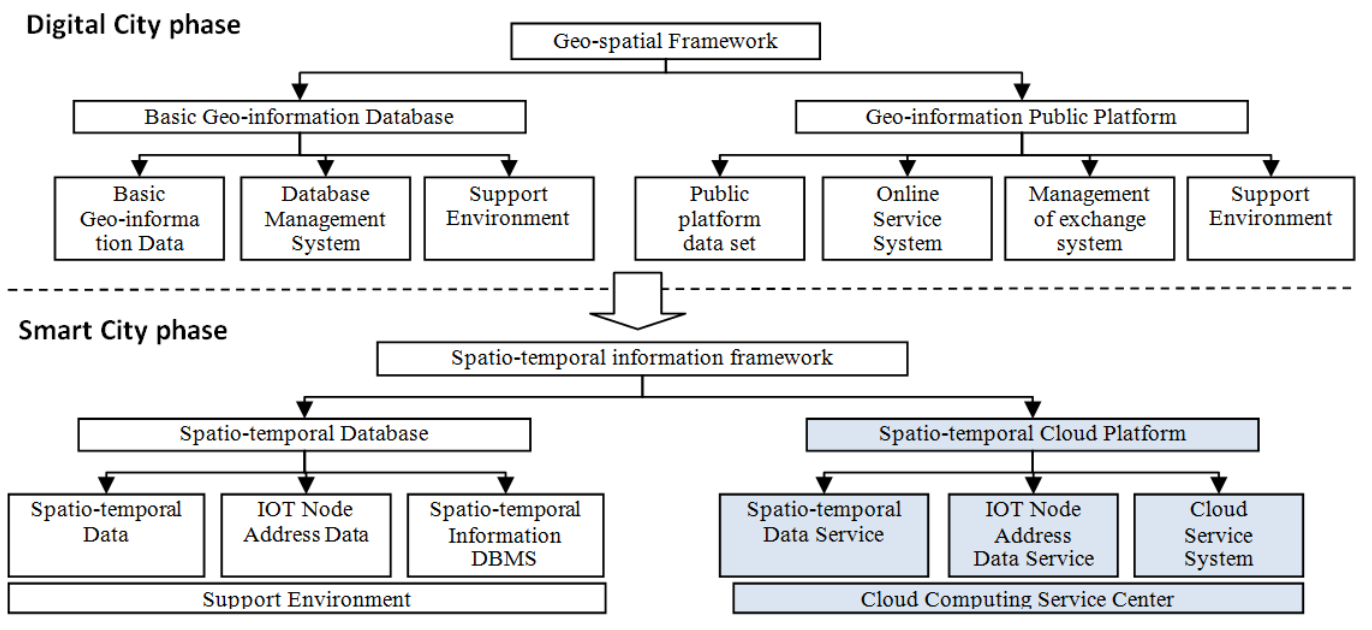

FIGURE I. DIGITAL CITY TO SMART CITY

\section{MAIN CONSTRUCTION CONTENTS AND KEY}

\section{TECHNOLOGIES OF SMG IN SMART CITY CONSTRUCTION}

In Smart City stage, facing the vast amounts of multi-source data, the user needs to extract valuable information analysis, simulation and prediction. Some units with limited basic conditions and needs expected through hardware hosting or construction, software unified development, and unified data management ways to saving resources and promoting application requirements. Up to now, the only authoritative SMG public platform, although reflects the common demand of each department, but the lack of customization capabilities. Smart City temporal cloud information platform is as basic platform which requires rich data, powerful function, providing spatial and temporal information support for the national economic construction and industrial applications. The overall structure is shown as in Figure II.

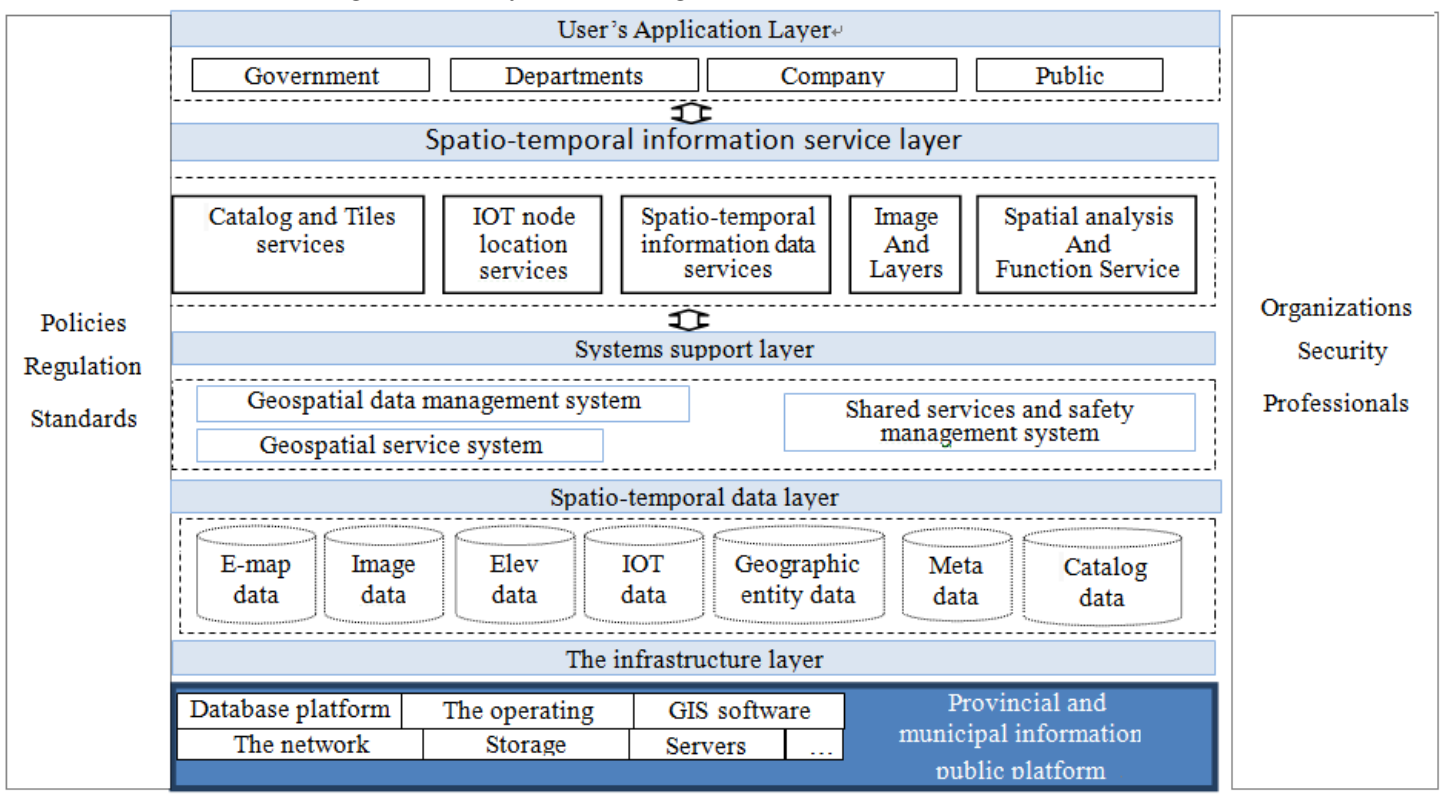

FIGURE II. TEMPORAL INFORMATION SMART CITY CLOUD PLATFORM ARCHITECTURE DIAGRAM 


\section{A. The Main construction Contents}

(1) The establishment of multi-temporal, multi-scale, multi-type, real-time spatial and temporal information database. Based on the basic geographic information database existing as digital city construction achievements, new information database will increase the intensity and frequency of data collection with temporal attributes, update basic geographic information data production, multi-sectoral thematic geography information and three-dimensional image data. Eventually, it will formed multi-scale, multi-type, high-quality urban space and time information database.

(2) The establishment of intelligently customize, server and host of space-time information cloud storage platform. Geographic information public platform is a common platform for all sectors, strong GIS department need to be more abundant, flexible, self-service approach, weak GIS departments need host Storage, easier, images, fast service. Thus, the platform need to increase spatial and temporal analysis, intelligent assembly, decision support, intelligent customization features such as host Storage, intelligent combination of knowledge engine driven, and providing services on demand.

(3) The establishment of policies, standards and mechanism related time and space information update, maintenance and use. For safe, efficient and convenient services, various types of data standards, technical specifications, interface specification, service standards, safety norms is essential, in addition, policy support, management system, updating and maintenance mechanisms are also very important.
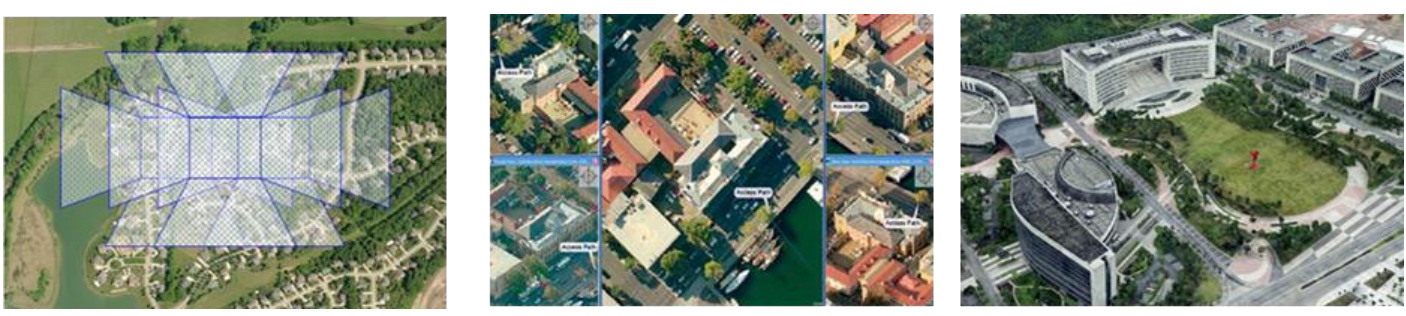

FIGURE III. CITY THREE-DIMENSIONAL MAP

2) Huge amounts data mining technology: Smart city construction, will inevitably produce huge amounts data which will be the treasure, and we need to be able to excavate its value $^{[2-5]}$. Data mining is a process of decision support, through which the highly automated analysis data could make the inductive reasoning, dig out the potential model and help policymakers adjust strategy, reduce risk and make the right decisions. The current means of access to information already quite rich. With the development of various industries, Smart City could provide basic geospatial information sharing and related geospatial statistical analysis, as well as through the effective integration of geospatial information and industry information with a variety of thematic statistical analysis methods (including space analysis), spatial modeling simulation forecasting further information. All these data and information could be deep analyzed through expert system for the further transformation of judgment and decision making. In
(4) Set up the application of the typical demonstration significance project. To demonstrate the application of Smart City is a comprehensive inspection. It is need to choose the applications which are close to the livelihood of the people, government concerned and good conditions for industry. Through these applications, the accumulation of experience, and constantly improve, and then gradually establish a spatial and temporal information cloud platform construction and service mode.

\section{B. Key Technology}

In recent years, many domestic experts and scholars in the field of Smart City technology continue to make breakthroughs $^{[1]}$. In SMG field, the technology remains the key and difficult as follows.

1) Multi-source data acquisition and fast processing technology: In recent years, technology development of domestic satellites, unmanned aerial vehicles and other rapid, high-resolution satellite remote sensing images and digital aerial digital images have become the main ways to obtain image data, but also is the main source of data in production of DOM, DLG, DEM, DSM for digital city. LiDAR system can be obtained by subsequent processing to facilitate data for City Model and vegetation; Oblique photography technology can quickly build three-dimensional model on the ground, more truly reflect the actual situation of ground objects. It is a new type of data resources, as shown in Figure III. All these provide a solid foundation for smart city construction. recent years, NASG puts forward many projects the government and people concerned, like geospatial framework construction for Digital City, the World Map building, National Geographic Conditions Monitoring etc. These hot issues related to livelihood, environmental, are carried out by making surveillance, basic statistics and integrated statistics. All above is positive and useful exploration about large amounts data mining.

3) Information data base and information engine: Information data base is an application knowledge base which consists of temporal data services, networking node localization services, development interface and map features, as well as registered professional features signature database. Information engine has a certain learning ability, and it could provide services on demand according to the key information provided by the user. When demand automatic assembly with establishing the adjustment man-machine cooperation 
environment, data deletions and interface tweaks improvements could be done according to inappropriate functions, data content and interface.

\section{SMG DOCKING WITH THE INFORMATIZATION}

The information construction of China starts relatively late, but developed rapidly in recent years. Many emerging industries and fields, such as communication, electronic commerce, etc. have reached a high level. The informationization development of SMG industry is overwhelming, especially means and capability in SMG data acquisition and expression. Industry informatization level is uneven between not only directly affect the internal information integration in the industry, at the same time will affect the information fusion between different management and industry, the emergence of Smart City will be to promote industry information fusion of a powerful propeller. For SMG industry, spatial information acquisition and offer ability are the foundation of the development condition. The most important factors is to find how connect SMG resources and other thematic information resources in the application. How to go better during Smart City construction, SMG needs steadily developing its own technology and mining and providing effective information related to other industries.

\section{CONCLUSION}

SMG industry developed from the earliest topographic map to $4 \mathrm{D}$ products and then to service oriented architecture of geographic information services, which has experienced great changes, but these are not adequate to meet the government, industry, and people's growing and changing needs. At present, SMG industry develop more variety of products, more powerful platform, and a more flexible service mode according to different industry requirements. SMG applications are blended with other industries to find the correspondence by digging deeper information fusion, modeling and simulation prediction methods.

Urbanization in China is a powerful driving force of smart city construction, there is no end in urban development, space and time information cloud platform constructions are also a process of dynamic, long and constantly improving. At the same time, smart city construction should move on step by step, extending from big cities to small cities and towns to rural area forming a seamless docking. Departments of SMG should position benchmark, resource reserves and supply, conquer technical problems such as statistical analysis, modeling, simulation, prediction etc. Moreover, they should further and perfect the standards for smart city construction and to provide the first-class service.

\section{REFERENCE}

[1] Winch, Ling-ling Li, Qing-jie Liu, et al. Vision-based high-resolution remote sensing images of notable and artificial segmentation of the target region extraction [J]. Surveying and Mapping, 2013,42 (06): 831-837.

[2] Jia-yao Wang. Big Data era of smart city [J]. Surveying and Mapping, 2014,39 (05): 3-7.

[3] Jing-nan Liu. Big data and location-based services [J]. Surveying and Mapping, 2014,39 (03): 3-9.
[4] Yong-Min Zhang. "Smart City" above the "digital city" [J]. China Information, 2011 (10): 12-17.

[5] Tang-tang Yang. From Digital City to study the wisdom of the city's building ideas and technical methods [J]. Geomatics World, 2013 (01): 63-67.

[6] Bing-zheng Gong. Thoughts on the Development of the wisdom of the city [J]. China Information, 2012 (11): 7-10. 